Results Response/learning from these events will be captured using film, questionnaires and focus groups and used to inform and update the hospice's dementia strategy. Key messages will be presented at conference with development of ongoing engagement and future research plans.

Conclusion Sharing experiences and displaying new, creative possibilities will aid understanding of those with barriers of memory and communication, unlocking gateways to connection with people living with dementia is possible.

\section{P-20 THINKING AHEAD CLINIC - EQUITABLE PALLIATIVE CARE FOR LIVER DISEASE PATIENTS}

Sarah Myford. Keech Hospice Care, Luton, Bedfordshire

10.1136/spcare-2021-Hospice.41

In early 2019 Keech Hospice Care was contacted by the consultant hepatologist at the local hospital who was concerned that liver patients were experiencing inequality of access to palliative care services. Due to the complexity of these patients they have high hospital attendances/admissions (over $70 \%$ of patients with advanced liver disease die in hospital), which could be prevented with increased shared care between the acute hospital and the hospice.

Two nurse practitioners from the hospice started working with the consultant hepatologist and commenced 'thinking ahead clinics'. A nurse practitioner joins the consultant in the hospital outpatient clinic to introduce hospice care, patients would then be invited to the hospice's nurse-led liver clinic.

The 'thinking ahead' clinic was initiated to talk about advance care planning, ensure the patient has access to all hospice services such as community support emotionally and physically; social work for help with finances, housing and will writing; carers support; talking therapies for emotional support; access to venepuncture, infusions and palliative doctor reviews, along with the rehabilitation team which facilitates keeping palliative patients well for as long as possible and our inpatient unit for symptom control or end-of-life care.

The community specialist teams accept referrals when there is complex physical or psychological needs. Until this time liver patients have limited access to services and support. By attending liver clinics we can ensure they have access to the right care throughout their illness so that plans are made before a crisis hits. This includes referring and working with community nursing teams, GPs, hospitals, the homeless and drug and alcohol services.

'The work we have been doing together, and the achievements are fantastic... an excellent example of what we can achieve by working together. I am very happy to know our patients have a better quality of life with our joint input' (Hepatology team: Acute hospital).

\section{P-21 A COLLABORATIVE INITIATIVE TO PROVIDE SUPPORT TO A MENTAL HEALTH UNIT DURING A COVID-19 OUTBREAK}

Anne Goggin, Kirsty Lewis. Salisbury NHS Foundation Trust, Salisbury, UK
Background In January 2021 there was an outbreak of COVID-19 at a local mental health unit. This adult unit with 67 beds cared for multiple psychiatric conditions. The appropriate transfer to the acute hospital of ill patients and support for the psychiatric team in managing those patients not for escalation was required.

Aims To promote equality of access for psychiatric inpatients who became ill with COVID-19 to appropriate management including from acute medicine and from palliative care.

Readily available palliative care support to medical and psychiatric teams regarding symptom control.

To enable decisions to be made re appropriately not escalating care to the acute hospital with the security of timely access to palliative care support.

Methods Establishment of a virtual ward round (VWR) at a set time daily attended by the psychiatric team, an Acute medical consultant and consultant or $\mathrm{SpR}$ in palliative care. Inclusion criteria were agreed for which patients to bring to the VWR. A treatment escalation plan was discussed for each patient.

Results VWR ran daily for three weeks and alternate days for a further two weeks. Twenty-five patients aged 20-80 years were referred to the VWR with a range of psychiatric conditions and medical co-morbidities. Twenty-three of the 25 were judged appropriate for escalation of treatment. Eight of the 23 were admitted to the acute hospital, two of whom died. For the remaining two patients the decision was made not for escalation, care to continue at MHU.

Conclusion The VWR provided a clear and timely process for referral and discussion of symptomatic patients. Treatment escalation plans were made in collaboration with the psychiatric team who knew the patients well, together with the expertise of acute medicine in the context of support from the palliative care service should the MHT need to care for those at end-of-life.

\section{P-22 PALLIATIVE CARE FOR ADULTS WITH NEURODISABILITY: PARTNERSHIP WORKING TO ACHIEVE SUSTAINABLE SUPPORT}

${ }^{1,2}$ Jo Elverson, ${ }^{1}$ Owen Lever, ${ }^{2}$ Kalyani Snell. 'St Oswald's Hospice, Newcastle upon Tyne, UK; ${ }^{2}$ Newcastle upon Tyne Hospitals NHS Trust, Newcastle upon Tyne, UK

\subsection{6/spcare-2021-Hospice.43}

Background Adults who have grown up with neurodisability experience multiple unmet needs (Solanke, Colver, McConachie, On behalf of the Transition collaborative group, 2018; PCPLD Network, NHS England, 2017). They are more likely to have unanticipated deaths, with limited opportunity for exploration of their holistic needs and wishes (Todd, Bernal, Shearn, et al., 2020), however, the unpredictable and long natural history of their condition makes it difficult to know when they would benefit most from palliative care input. Few adult palliative care services can sustain support to patients with a complex but stable condition who may live for many years.

Aims We explore a new model of collaborative working to sustainably address the unmet palliative care needs in this patient group. By regularly meeting with specialist clinicians from neuro-rehabiliation, respiratory medicine, intensive care, neurology, primary care and gastroenterology, palliative care specialists aim to: 